\title{
Global search optimization using the particle swarm algorithm applied to photoelectron diffraction
}

\section{Wellington D. Pereira*, Luis H. de Lima, Abner de Siervo}

\begin{abstract}
The photoelectron diffraction (PED) is an element and chemical selective technique, which have been used for surface structure determinations. Due the complexity phenomena of low-energy photoelectron diffraction, obtain the structure by holographic inversion method from the experimental data present difficulties which unfeasible the method. To perform a structure determination It $s$ necessary use the comparative method between the experimental and theoretical data from a hypothetical structure in a try-and-error approach, which could spent high computational time. In this project we have developed a Particle Swarm Optimization (PSO) algorithm for structural determination using the PED technique.
\end{abstract}

\section{Key words:}

Photoelectron diffraction, Swarm optimization, surface structure

\section{Introduction}

The PED is based on the photoelectric effect where a photoelectron is ejected from an atom by absorbing an x-ray photon. During the transport of the photoelectron from the emitter atom to the electron detector it can be scattered by the electrostatic potential of the neighbor atoms that produces an interference pattern, a PED pattern, which brings information about the atomic structure surrounding the emitter [1]. To proper determine the surface structure that has generated the PED pattern, it is necessary to theoretically simulate such a pattern and then compare it with the experimental data. Many groups in the world use the Multiple Scattering Calculation of Diffraction (MSCD) package for PED simulation [2].

To optimize the procedure for the surface structure determination, the PSO code was developed and integrated with MSCD. Technically, PSO can be split in two branches, the global and local topology, which have been extensively studied in literature. In this project we have implemented a hybrid topology (HPSO) $[3,4]$ that consists in changing from local to global topology, with the purpose to search for global minimum and improve the convergence rate.

\section{Results and Discussion}

The HPSO code developed was compared with others PSO available in the literature using the Rastrigin Function, the benchmark is below:

\section{Image 1. Rastrigin}

$$
\text { Function }
$$

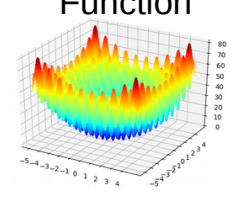

Table 1. Benchmark PSO

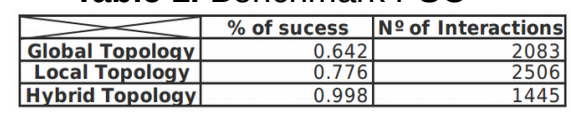

Table 1. show the high quality of HPSO when compared with others topologies of PSO. The HPSO provide a really great rate of success aligned with the best convergence rate, optimizing the function using the lowest number of interactions.
After the HPSO development and its integration with the MSCD code, we started to do the surface structure determination of real systems such as a $\mathrm{MoTe}_{2}-$ (0001) crystal. The theoretical and experimental pattern is showed below:
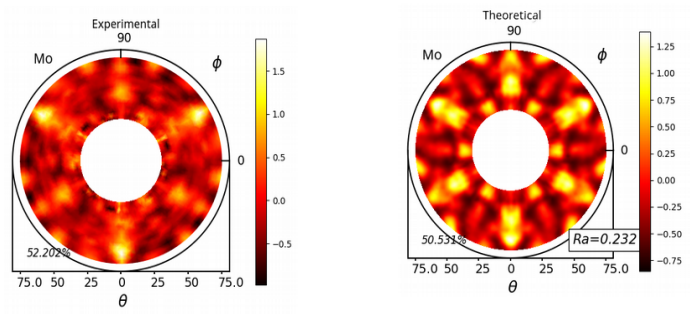

The visual comparison of the two patterns show to us that the code converge to the right structure, however the quantitative comparison indicates that theoretical structure is not perfect yet.

\section{Conclusions}

The search method using particle swarm returned good results, demonstrating high performance in determining the best surface structural parameters in accordance with the real structure of $\mathrm{MoTe}_{2}-(0001)$ crystal, in a reduced computational time.

Furthermore, the hybrid technique of swarm particle present superior results when compared with traditional swarm particle, providing a superior rate of success, at the same time is capable to converge faster than others topologies.

\section{Acknowledgement}

This work was financially supported by FAPESP (2016/21402-8 and 2017/18574-4), CAPES and CNPq (455807/2014-0) from Brazil. WDP gratefully acknowledge PIBIC-CNPq for the studentship.

\footnotetext{
${ }^{1}$ de Siervo, A. ; Lima, L. H., Difração de Fotoelétrons (2017)

${ }^{2}$ http://www.ap.cityu.edu.hk/SurfStrucInfo/SurfStrucInfo_files/mscd/mscdover.html

${ }^{3}$ Marinakis,Y.; Eur. J. of Op. Res. 2017, 261, 819.

${ }^{4}$ Abraham, A.; Liu, H.; Chang, T.; Gen. and Evol. Comp. Conf. 2016, 197204.
} 